\title{
Escherichia coli Nissle 1917 secreting functional interleukin 2 targets tumours and enhances the immune response to suppress tumours
}

Binghua Lu

Hunan Normal University

Huijun Yang

Hunan Normal University

Fei Liu

Hunan Normal University

Yunjun Sun

Hunan Normal University

Haocheng He

Hunan Normal University

Xuezhi Ding

Hunan Normal University

Shengbiao Hu

Hunan Normal University

liqiu xia ( $\nabla$ xialq@hunnu.edu.cn )

Hunan Normal University https://orcid.org/0000-0001-8443-2916

\section{Research}

Keywords: E.coli Nissle 1917, IL-2, Anti-tumor engineering bacteria, Targeted cancer therapy

Posted Date: May 11th, 2020

DOI: https://doi.org/10.21203/rs.3.rs-27294/v1

License: (c) (1) This work is licensed under a Creative Commons Attribution 4.0 International License. Read Full License 


\section{Abstract}

Escherichia coli Nissle 1917 (EcN) is non-pathogenic probiotic bacteria. Previous studies have indicated that $\mathrm{EcN}$ can accumulate and proliferate selectively in solid tumours in BALB/c mouse models. In this study, EcN was engineered to express human interleukin 2 (hIL-2), which is known to enhance immune responses to tumours by activating a variety of immune cells. IL-2 expressed by EcN was proven to activate PBMCs in vitro. Compared to control EcN, intraperitoneally injected EcN expressing hIL-2 (EcN(hIL-2)) was selectively distributed in the tumour microenvironment and inhibited the growth of CT26 tumours in a tumour-bearing mouse model. Antitumour activity was achieved without toxicity to key normal organs and tissues, such as liver, spleen and kidneys. The antitumour mechanism was associated with the infiltration of inflammatory cells, such as T cells, neutrophils and macrophages. These findings provide evidence that the combination of tumour-targeting EcN bacteria and delivery of the immunostimulatory factor IL-2 can be exploited as a promising tumour immunotherapy.

\section{Introduction:}

Immune system function is often inhibited in the microenvironment of solid tumours[1-5]. Therefore, many attempts are being made to activate immune responses against tumours[6]. Tumour-targeted therapy is a promising treatment method for many malignant tumours. Previous studies have indicated that systematically administered Escherichia coli Nissle 1917(EcN) can accumulate and proliferate selectively in the interface between the viable and necrotic regions of solid tumours[7]. The immunoregulatory cytokine interleukin-2 (IL-2) is a growth and activating factor for a variety of immune cells, including T cells and NK cells[8, 9]. It can promote the production of NK or T cell-derived cytokines, such as TNF-a, IFN-y and GM-CSF, which can boost antitumour immunity, and these molecules have synergistic effects[10-12]. Therefore, we hypothesized that tumour-targeting bacteria expressing the immune-activating cytokine IL-2 may optimally modify the immune microenvironment and improve antitumour effects.

In this study, EcN bacteria were engineered to express soluble human IL-2, with the aim of improving the immune function of tumour-bearing mice and inhibiting the growth of tumours. To exploit the role of IL-2 delivered by EcN in tumour tissue, CT26 colon cancer cells were implanted subcutaneously in syngeneic $\mathrm{BALB} / \mathrm{c}$ mice, and after the tumour was established, $\mathrm{EcN}(\mathrm{hlL}-2)$ was injected intraperitoneally into the tumour-bearing mouse. Immunohistochemical results showed that EcN(hIL-2) specifically localized in the tumour region and that IL-2 was released in the tumour tissue. Tumour growth in the EcN(hIL-2) group was inhibited approximately $53.91 \%$ compared with that in the PBS control group. A myriad of necrotic tumour cells could be observed in the tumour tissue of the EcN (hIL-2)-treated group. To assess the toxicity induced by EcN (hIL-2), we measured the body weight of tumour-bearing mice every two days. These mice were sacrificed after 7 days, and the liver, kidneys and the spleen of the mice in each group were excised and weighed. There was no difference in either body or organ weight between the experimental and control groups. We also investigated the distribution of engineered bacteria in the liver, 
kidneys and the spleen of tumour-bearing mice using an IVIS spectrum[13,14] and found that the numbers of bacteria in these organs were significantly lower than those in tumours.

To further explore the antitumour mechanism of these engineered bacteria, local immune responses induced by EcN(hIL-2) were also detected by H\&E staining of tissue sections. Immune cells, such as T lymphocytes, neutrophils and macrophages, infiltrated the tumour microenvironment, but the control groups had insufficient immune cell infiltration. We also examined the cytokines IFN- $\gamma$ and TGF- $\beta$ in the serum, as IFN- $\gamma$ is a known key mediator of IL-2 toxicity and TGF- $\beta$ is an inhibitory factor. The results showed that EcN(hIL-2) treatment was associated with significantly elevated IFN-y expression and decreased TGF- $\beta$ expression.

\section{Results}

\section{Expression analysis of the IL-2 protein in vitro}

To allow expression of the IL-2 protein in a prokaryotic system, the IL-2 gene was cloned into three different inducible expression vectors (Supplementary Fig. 1A, 1B and 1 C). The differential proteins expressed by the three recombinant plasmids in E. coli BL21 (DE3) were verified by Western blotting and mass spectrometry, which proved that the target protein IL-2 was successfully expressed (Supplementary Fig. 2 and Table S1). Coomassie Brilliant Blue staining showed that the recombinant protein showed soluble expression under the action of SUMO and IF2 tags (Fig. 1A). Engineered bacteria need to continuously secrete recombinant proteins inside a tumour, induce immune cell activation, attack tumour cells, and inhibit tumour growth. Therefore, the IL-2 protein can be continuously expressed in hypoxic tumours under the oxygen-dependent promoter of the haemoglobin gene (vhb) of Vitreoscilla and the pelB leader sequence (Fig. 1B and Supplementary Fig. 3). Engineered bacteria were cultured overnight in LB medium, and the recombinant protein was successfully expressed in engineered EcN bacteria, as verified by SDS-PAGE analysis (Fig. 1C). Western blot analysis indicated that the IL-2 protein was presented in both the cell lysate and medium supernatant of EcN (hIL-2) (Fig. 1D). To reduce the effect of tags on IL-2 protein activity, a SOMO fusion system with a relatively low molecular weight was selected for subsequent experimental research.

\section{SUMO-IL-2 protein can promote the proliferation of PBMCs}

BL21 (pSmartl-IL 2) bacteria were cultured, and IL-2 protein was collected by Ni-NTA Sefinose (TM) Resin Kit. The IL-2 protein at different concentrations was cocultured with peripheral blood mononuclear cells (PBMCs), and PBS was added to the control group. PBMCs were cocultured with the recombinant protein for $24 \mathrm{~h}$, and then Cell Counting Kit-8 was added to detect the cell survival rate. The results showed that cell proliferation was obviously promoted after the addition of SUMO-IL-2 (Sumo is the solubilizing label on pSmartl), and the proliferation rate of cells also increased as the SUMO-IL-2 protein concentration increased (Fig. 2A). Additionally, the cell culture medium was centrifuged after the incubation, and the culture supernatant was collected. The concentrations of IFN- $y$ and TGF- $\beta$ in the supernatant of the 
culture medium were detected by enzyme-linked immunosorbent assay (ELISA). The results showed that compared with that in the supernatant of the control group, the concentration of IFN-y in the supernatant of the experimental group was significantly increased (Fig. 2B), while the concentration of TGF- $\beta$ was significantly decreased (Fig. 2C).

\section{EcN specifically colonizes tumour regions in tumour- bearing mice}

An IVIS can accurately observe the real-time location of bacteria in animals without causing damage to the animals[13, 14]. After intraperitoneal injection of $5 \times 10^{6} \mathrm{CFU} / 100 \mu \mathrm{L} \mathrm{ECN(Lux)} \mathrm{into} \mathrm{tumour-bearing}$ mice in the experimental group and injection of sterile PBS into tumour-bearing mice in the control group, bacterial colonization in the mice was observed by an IVIS. The results showed that the tumour-bearing mice exhibited a significant fluorescence signal in the tumour area for 5 days after the bacteria were injected, while the control group did not exhibited a detectable signal, and the fluorescence signal was still observed on the 7th day after injection (Fig. 3A). After the mice were euthanized on the 7th day, the tumour, liver, kidneys and spleen of the mice were obtained. IVIS analysis showed that 5 days after $\mathrm{EcN}$ (Lux) was intraperitoneally injected into tumour-bearing mice, a strong fluorescence signal was detected in the tumour tissues of the mice, and no fluorescence signal was detected in other organs (Fig. 3B). These results showed that EcN has excellent targeting to the tumours in CT26 tumour-bearing mice. Bacteria can quickly accumulate in the tumour area and grow and reproduce in the tumour area after intraperitoneal injection into mice, while bacteria in other parts of mice can be removed by the local immune response quickly.

\section{Antitumour effect of EcN(hIL-2)}

To validate the successful expression of the IL-2 molecule carried by EcN in tumour areas, we performed immunohistochemistry on samples from each group of mouse tumours. Mice were euthanized on the 7th day after the third administration, and the tumour tissue was removed, fixed in $4 \%$ paraformaldehyde, and then embedded in paraffin. These results showed that a yellow-grey signal appeared in the tumour tissue sections of the EcN (hIL-2) experimental group, while those of the other three groups did not show a positive signal (Fig. 4A). These results indicate that IL-2 is successfully expressed in the tumour region.

To evaluate the antitumour efficacy of EcN (IL-2), we subcutaneously injected CT26 colon cancer cells into the right axillary area of BALB/c mice. The resultant xenograft tumour model was used to study the antitumour effect of EcN (hIL-2). When the tumours in the mice grew to approximately $60 \mathrm{~mm}^{3}$, the mice were randomly divided into 4 groups $(n=5,6$, or 7$)$, and the groups were treated respectively by intraperitoneal injection of sterile PBS, EcN, EcN (28a) or EcN (hIL-2). Body weight and tumour volume were measured every two days during the observation period until the animals were sacrificed. The results of the experiment showed that the tumour growth in the EcN (hIL-2) group was significantly inhibited (Fig. 4B), while the xenograft tumour growth in the other three groups have no difference. The final tumour weight of the EcN (hIL-2) group was also significantly lower than that of the other 3 groups 
(Fig. 4C). The tumour volume in the PBS group reached $4.93 \pm 1.35 \mathrm{~cm}^{3}$, but the tumour volume in the EcN (hIL-2) group was significantly smaller, reaching a volume of $2.32 \pm 1.43 \mathrm{~mm}^{3}$. Tumour volume was calculated according to the formula (Table 1). Tumour growth in the EcN (hIL-2) group was inhibited approximately $53.91 \%$ compare to that in the PBS group.

Table 1

The comparison of tumor volume, tumor weight of CT26 cancer of the $\mathrm{BALB} / \mathrm{c}$ mice

\begin{tabular}{|lll|}
\hline Group & Mean Tumor Volume $\left.\mathbf{( c m}^{3}\right)$ & Mean Tumor Weight (g) \\
\hline PBS & $4.93 \pm 1.35$ & $6.41 \pm 1.22$ \\
\hline EcN & $5.53 \pm 1.43$ & $6.74 \pm 1.35$ \\
\hline EcN (28a) & $4.62 \pm 1.67$ & $6.38 \pm 0.91$ \\
\hline EcN (IL-2) & $2.32 \pm 1.43(53.91 \%)^{\star \star}$ & $3.48 \pm 1.49(45.71 \%)^{*}$ \\
\hline
\end{tabular}

Tumour histomorphology and safety monitoring of E. coli Nissle 1917

Haematoxylin-eosin staining (H\&E staining) is one of the commonly used staining methods for paraffin sections. Haematoxylin dyeing solutions are alkaline, mainly causing chromatin in the nucleus and nucleic acid in the cytoplasm to be stained purple-blue; eosin is an acidic dye, which mainly stains the components in the cytoplasm and the extracellular matrix red. Our experimental results showed that the tumour staining results in the PBS group showed normal tumour cell morphology, and no necrotic areas were observed. However, infiltrating inflammatory cells were observed in the EcN experimental group, and the cell morphology was irregular (Fig. 5A).

Additionally, we also performed H\&E staining of liver, kidney and spleen tissues from the PBS group and $\mathrm{EcN}$ experimental group. The results showed that there was no significant change in histopathological morphology in the liver, kidneys or spleen between the two groups, indicating that $\mathrm{ECN}$ had no obvious side effects on the liver, kidneys or spleen in mice (Fig. 5A). During the experiment, to assess the systemic effects of EcN on the whole body after intraperitoneal injection, we measured mouse body weight every two days. At the end of the experiment, the liver, kidneys and spleen of the mice in each group were excised and weighed, and there were no differences in weight among the four groups (Fig. 5B). Although the mice exhibited a slight decrease in body weight during treatment, they all approached the same weight by the end of the experiment (Fig. 5C). All of the above results demonstrate that the toxicity of intraperitoneal administration of $\mathrm{EcN}$ to mice is negligible.

\section{Examining the tumour microenvironment}

We explored the mechanism underlying the antitumour immune activity of EcN (hIL-2) in a CT26 colon cancer tumour model. We investigated the immune cell profile in the tumour microenvironment and the changes in cytokines in the blood using the CT26 tumour model. We selected specific antigens to be 
combined with antigens on the surface of T lymphocytes, neutrophils and M1 macrophages, and compared the changes in the content of these three cells in the four groups of tumors by immunohistochemistry. Immunohistochemical results showed that the levels of tumour-infiltrating T lymphocytes and neutrophils were increased in the EcN (hIL-2)-treated group compared with the other three groups (Fig. 6A, B). The results also showed an increase in the level of M1 macrophages in the total macrophage population in the tumour microenvironment after treatment with EcN (hIL-2) (Fig. 6C). Next, we examined the levels of two immune factors in the blood in four groups of mice. The data showed that the IFN- $\gamma$ level was significantly increased and the TGF- $\beta$ level was decreased in the blood of mice in the EcN (hIL-2)-treated group compared with that of mice in the other experimental groups (Fig. 6D, E). Collectively, these data indicate that EcN (hIL-2) treatment improves the immune microenvironment of tumour-bearing mice to some extent, leading to improved survival outcomes after EcN (hIL-2) treatment.

\section{Discussion:}

Tumour-targeted therapies and immunotherapy have raised hope for curing many malignant cancers[15]. Live tumour-targeting bacteria are a distinctive option for tumour therapy. Bacterial vectors can be reprogrammed following simple genetic rules or sophisticated synthetic bioengineering principles to produce and deliver antitumour agents based on clinical needs. Attenuated Salmonella typhimurium, Clostridium novyi, Bifidobacterium and Listeria strains have been tested in animal models and have shown preferential targeting of solid tumours, and several of these strains have advanced to clinical trials[16-20]. Various therapeutic payloads delivered by these tumour-targeting bacteria have since been developed [21-23]. However, these strains are all pathogenic bacteria, and systemic toxicity limits their clinical use. Although researchers have been focused on attenuating the virulence of these bacteria, there are many challenges.

Escherichia coli Nissle $1917(\mathrm{EcN})$ is known to be avirulent and consumed as the probiotic preparation Mutaflor, which is used for the treatment of various intestinal disorders; $\mathrm{EcN}$ successfully colonizes the human gut and can survive and proliferate in both hypoxic and oxygenated environments[24, 25]. Previous studies have demonstrated that $\mathrm{EcN}$ has excellent performance in preferentially localizing to tumours when administered systematically or orally to different tumour-bearing mouse models[7, 26]. $\mathrm{EcN}$ has also been engineered to deliver various antitumour agents and displays significant tumoursuppressive effects[26, 27]'[28].

IL-2 is a monomeric secreted glycoprotein with a molecular weight of $15 \mathrm{kDa}$ that exerts a wide spectrum of effects on the immune system and plays crucial roles in regulating both immune activation and homeostasis[12]. It was approved for use in clinical cancer immunotherapy several years ago[29]. However, high-dose IL-2 administration can result in severe systemic toxicity, such as malaise, fever, anasarca, jaundice, renal dysfunction and capillary leak syndrome, in many patients[30, 31]. Researchers have attempted to diminish the adverse effects of systemically administered IL-2 by altering the dose, schedule and route of administration[32-36]. Nevertheless, the toxic effects of IL-2 therapy persist to various degrees[37]. These shortcomings have made it necessary to create a better method of using IL-2 
for tumour therapy. Localized administration delivered by tumour-targeting bacteria is an appropriate option. In an attempt to establish a local delivery system for IL-2 that may diminish or prevent side effects, Denial A and colleagues used attenuated Salmonella typhimurium to produce the human IL-2 protein and significantly reduced the hepatic metastasis of colon cancer through gavage feeding of their engineered bacteria to model mice[38, 39].

Based on the current understanding of the tumour microenvironment and recombinant DNA technology, in this study, we addressed three major questions. First, engineered EcN expressing IL-2 can target CT26 in model mice. Second, IL-2 can enhance immune responses in the tumour microenvironment. Last, improved immune responses can disturb tumour tissues and suppress tumour growth. To achieve sustained high levels of IL-2 in the tumour microenvironment while avoiding systemic toxicity, we utilized the oxygen-dependent promoter of the haemoglobin gene $(\mathrm{vhb})$ of Vitreoscilla and the pelB leader sequence to facilitate IL-2 expression in the hypoxic tumour region. These engineered bacteria containing IL-2 are similar to a vaccine against tumours, and this vaccination strategy does not require knowledge of tumour antigens. Therefore, this approach may have advantages over nonimmunogenic approaches.

Taken together, our data demonstrate that the tumour-targeted bacteria EcN can express soluble hIL-2, localize in solid tumours and elicit local immune responses that induce tumour suppression while avoiding systemic toxicity. This live vector system is relatively inexpensive and does not require vast laboratory resources to produce antitumour reagents. However, the clinical development of live bacteria as therapeutic agents faces substantial hurdles mainly because of potential infection-associated toxicities, especially when administered systematically. Major efforts should be made to develop proper administration routes that can minimize systemic toxicities. Employing an oral route of administration in a syngeneic, xenograft CT26 colon cancer mouse model and exploring the cellular and immunological mechanisms of how EcN(hIL-2) facilitates the inhibition of tumours are the subjects of our ongoing research.

\section{Materials And Methods}

\section{Animals and cell culture}

All animal experiments followed the National Institutes of Health Guide for the Care and Use of Laboratory Animals and were approved by the Animal Ethics Committee of Hunan Normal University. Specific pathogen-free (SPF), male BALB/c mice were purchased from the SLRC Laboratory Animal Company (Hunan, China), and used at 6-8 weeks of age. CT26 colon carcinoma lines were maintained by our laboratory, and peripheral blood mononuclear cells (PBMCs) were purchased from Allcells Biotechnology (Shanghai) Co., Ltd.

CT26 cell suspensions were seeded in a cell culture dish, and $8 \mathrm{~mL}$ RPMI- 1640 medium containing 10\% FBS was added to resuspend the cells, which were cultured at $37^{\circ} \mathrm{C}$ in a $5 \% \mathrm{CO}_{2}$ incubator. The cell culture medium was changed every day. PBMCs frozen in liquid nitrogen were thawed and resuspended 
in RPMI-1640 medium containing $10 \%$ FBS at a final concentration of $5 \times 10^{6}$. The PBMCs were inoculated into 96-well plates, and different concentrations of recombinant protein were added. After $48 \mathrm{~h}$ of incubation at $37^{\circ} \mathrm{C}$ in a $5 \% \mathrm{CO}_{2}$ incubator, Cell Counting Kit-8 (CCK-8) was used for detection. CT26 cells are stored in this laboratory.

\section{Gene cloning and soluble expression of IL-2}

E. coli bacteria (pINCY-IL-2) were a gift from Li Qing in Wuhan. The primers used in the study are listed in Supplementary Table 2. IL-2 was cloned into the BamH I and Hind III restriction sites of pET- 28a, pSmart-I (a small ubiquitin-related modifier-SUMO fusion expression system) and pSmart II (an initiation factor-IF2 protein structure domain I fusion expression system). The three plasmids (pET-28a-IL 2, pSmart-IIL 2, and pSmart-II-IL 2) were transformed into E. coli BL21(DE3) cells, cultured at $37^{\circ} \mathrm{C}$ and $220 \mathrm{rpm}$ overnight, and transferred into $2 \%$ LB medium supplemented with $50 \mu \mathrm{g} / \mathrm{ml}$ kanamycin. Until the OD600 value reached 0.4-0.6, IPTG (final concentration of $0.4-0.5 \mu \mathrm{g} / \mathrm{mL}$ ) was added, and incubation was performed for $120 \mathrm{~min}$ at $30^{\circ} \mathrm{C}$. The bacteria-inducing culture solution was placed in an EP tube and centrifuged at $9000 \mathrm{rpm}$ for $3 \mathrm{~min}$ to collect the cells. After washing twice and resuspension in a mixture of $50 \mathrm{mM}$ $\mathrm{NaH}_{2} \mathrm{PO}_{4}$ and $300 \mathrm{mM} \mathrm{NaCl}$ at pH 8.0, the cells were lysed by sonication, and the supernatants and pellets were analysed by sodium dodecyl sulfate-polyacrylamide gel electrophoresis (SDS-PAGE) after centrifugation. The recombinant protein was cut out of the gel with a scalpel and identified by LTQ XL mass spectrometry (Thermo Fisher) after proteolysis.

\section{Construction of EcN expression strains}

The Vitreoscilla haemoglobin gene promoter Pvhb was amplified from pET-28a-Pvhb-pelB-asp (Lab Store). In addition, the Sumo-IL 2 fragment was amplified from pSmart-IIL 2 (constructed in this study). Pvhb-pelBSUMO-IL 2 was obtained by overlap extension PCR and inserted into pET-28a after digestion by Apa I and Hind III. The sequenced vector was transformed into EcN, which was then named EcN (hIL-2).

\section{Tumour inoculation and animal studies}

Animals were quarantined for 1 week prior to their use in the study. For the colorectal tumour model, $1 \times$ $10^{5} \mathrm{CT} 26$ cancer cells suspended in $100 \mu \mathrm{L}$ PBS were injected subcutaneously into the right axillary region of $B A L B / c$ mice.

After the tumour volume reached approximately $60 \mathrm{~mm}^{3}$, the mice were randomly divided into 4 groups (5-7 mice per group). EcN, EcN (28a), and EcN (IL 2) were activated overnight. For intraperitoneal injection of sterile PBS, EcN, EcN (28a) or EcN (IL 2) into the mice, the number of bacteria injected was 5 $\times 10^{6}$ colony forming units (CFU)/ $100 \mu \mathrm{L}$, and the injections were performed once every 7 days, for a total of 3 injections. Then, the mice were sacrificed and analysed on the fourteenth day, and the tumour, liver, kidney, and spleen weights of the mice were measured after the end of the experiment. Tumour volumes were determined by measuring two perpendicular diameters with a calliper according to the formula volume $=\left(a \times b^{2}\right) / 2$, where $a$ is the largest dimension and $b$ is the smallest dimension of the tumour. Body weight and tumour volume were measured every 2 days over the whole experiment. The antitumour 
activities of the treatments were evaluated by monitoring tumour growth inhibition. The tumour suppression percentage was calculated by the following computational formula: (control group-treatment group) / control group $\times 100 \%$ (with tumour volume or tumour weight used for calculations).

\section{Non-invasive in vivo imaging}

The bacterial distribution was monitored in injected mice. After 10 days of modelling in tumour-bearing mice, when the tumours of the mice had grown to approximately $100 \mathrm{~mm}^{3}$, the mice were intraperitoneally (i.p.) injected with $5 \times 10^{6} \mathrm{CFU} / 100 \mu \mathrm{L} \mathrm{ECN} \mathrm{(Lux)} \mathrm{(the} \mathrm{plasmid} \mathrm{is} \mathrm{preserved} \mathrm{in} \mathrm{our}$ laboratory) to observe the colonization of living animals by the bacteria at different time points using an in vivo imaging system (IVIS; Calipers). The mice were anaesthetized with $2 \%$ isoflurane by using an XGI8 gas system (Calipers). The mice were sacrificed 1 day or 7 days after the injection of EcN (Lux), and the tumour, liver, kidneys and spleen of the mice were dissected. The distribution of bacteria in each tissue was observed by IVIS.

\section{Histological morphology and the tumour microenvironment}

Tumours isolated from mice after sacrifice were placed in $4 \%$ paraformaldehyde overnight and then embedded in paraffin. Then, the tumours were prepared for haematoxylin and eosin staining and immunohistochemical staining assays in accordance with standard laboratory procedures. The expression of IL-2 and changes in several important immune factors within the tumour microenvironment were analysed by immunohistochemical staining. The liver, spleen, and kidneys of the mice in the PBS group and EcN groups were also prepared for H\&E staining to determine whether EcN has noticeable toxicity to mice.

After the cell experiment and the mouse experiment, the cell culture supernatant and mouse serum were separately collected, and $\gamma$-interferon (IFN- $\gamma$ ) and transforming growth factor- $\beta$ (TGF- $\beta$ ) levels were measured by ELISA.

\section{Statistics}

All data are expressed as the mean \pm standard deviation and were analysed using IBM SPSS statistics 21.0 software. Statistical analyses were performed using an unpaired Student's t-test. Differences with $P$ values less than 0.05 were considered to be statistically significant, whereas $P<0.01$ was considered to be very significant.

\section{Abbreviations}




\begin{tabular}{|ll|}
\hline bp & Base pair \\
\hline CCK-8 & Cell Counting Kit-8 \\
\hline OD & Opitical Density \\
\hline LD & linear dichroism \\
\hline PBS & Phosphate-buffered saline \\
\hline PCR & Polymerase chain reaction \\
\hline LC-MS/MS & Liquid chromatography-tandem mass spectrometry \\
\hline EDTA & Ethylene diamine tetraacetic acid \\
\hline SDS-PAGE & SDS-poly acrylamidegel electrophoresis \\
\hline Tris & Tris (hydroxymethyl) aminomethane \\
\hline kDa & kiloDalton \\
\hline H\&E & Hematoxylin-eosin stainin \\
\hline IHC & Immunohistochemistry \\
\hline E.coli & Escherichia coli \\
\hline EcN & E.coli Nissle 1917 \\
\hline IL-2 & Interleukin-2 \\
\hline ELISA & enzyme linked immunosorbent assay \\
\hline
\end{tabular}

\section{Declarations}

\section{Acknowledgements}

Not applicable.

\section{Funding}

This work was supported by the National Natural Science Foundation of China (31770106),the National Basic Research Program (973) of China (2012CB722301), the International Cooperation Project (0102011DFA32610) and the Cooperative Innovation Center of Engineering and New Products for Developmental Biology of Hunan Province (20134486).

\section{Author Contributions:}


Conceived the research idea: BHL, LQX, HJY. Designed the experiments: BHL, HJY, LQX, YJS.

Methodology: BHL, FL, XZD, HCH. Performed the experiments: BHL, HJY, HCH. Discussed the results: BHL, HJY, LQX. Analyzed the data: BHL, HJY, SBH. Wrote the manuscript: BHL,HJY.

Discussed and revised the manuscript: BHL, HJY, HCH, YJS, XZD, LQX. All authors read and approved the final manuscript.

\section{Availability of data and materials}

All data generated or analysed during this study are included in thispublished article and its supplementary information files.

\section{Ethics approval and consent to participate}

Not applicable.

\section{Consent for publication}

Not applicable.

\section{Conflict of interest}

The authors declare that they have no conflict of interest.

\section{Author details}

${ }^{1}$ Hunan Provincial Key Laboratory of Microbial Molecular Biology, State Key Laboratory of Developmental Biology of Freshwater Fish, College of Life Science, Hunan Normal University, Changsha 410081, People's Republic of China..

\section{References}

1. Mlecnik B, Bindea G, Kirilovsky A, Angell HK, Obenauf AC, Tosolini M, et al. The tumor microenvironment and Immunoscore are critical determinants of dissemination to distant metastasis. Science translational medicine. 2016;8(327):327ra326.

2. Oluwadara O, Giacomelli L, Brant X, Christensen R, Avezova R, Kossan G, et al. The role of the microenvironment in tumor immune surveillance. Bioinformation. 2011;5(7):285-90.

3. Peinado H, Lavotshkin S, Lyden D. The secreted factors responsible for pre-metastatic niche formation: old sayings and new thoughts. Sem Cancer Biol. 2011;21(2):139-46.

4. Chen DS, Mellman I. Oncology meets immunology: the cancer-immunity cycle. Immunity. 2013;39(1):1-10.

5. Spranger S. Mechanisms of tumor escape in the context of the T-cell-inflamed and the non-T-cellinflamed tumor microenvironment. Int Immunol. 2016;28(8):383-91. 
6. Yang $B$, Wang $\mathrm{C}$, Xie H, Wang $\mathrm{Y}$, Huang J, Rong Y, et al. MicroRNA-3163 targets ADAM-17 and enhances the sensitivity of hepatocellular carcinoma cells to molecular targeted agents. Cell death disease. 2019;10(10):784.

7. Stritzker J, Weibel S, Hill PJ, Oelschlaeger TA, Goebel W, Szalay AA. Tumor-specific colonization, tissue distribution, and gene induction by probiotic Escherichia coli Nissle 1917 in live mice. International journal of medical microbiology: IJMM. 2007;297(3):151-62.

8. Liao W, Lin JX, Leonard WJ. Interleukin-2 at the crossroads of effector responses, tolerance, and immunotherapy. Immunity. 2013;38(1):13-25.

9. Lotze MT, Matory YL, Ettinghausen SE, Rayner AA, Sharrow So, Seipp CA, et al. In vivo administration of purified human interleukin 2. II. Half life, immunologic effects, and expansion of peripheral lymphoid cells in vivo with recombinant IL 2. Journal of immunology. 1985;135(4):2865-75.

10. Waldmann TA. The IL-2/IL-2 receptor system: a target for rational immune intervention. Immunology today. $1993 ; 14(6): 264-70$.

11. Sun $Q$, Zhang $X$, Wang $L$, Gao X, Xiong $Y$, Liu L, et al. T-cell receptor gene therapy targeting melanoma-associated antigen-A4 by silencing of endogenous TCR inhibits tumor growth in mice and human. Cell death disease. 2019;10(7):475.

12. Boyman 0 , Sprent J. The role of interleukin-2 during homeostasis and activation of the immune system. Nature reviews Immunology. 2012;12(3):180-90.

13. Danino T, Prindle A, Kwong GA, Skalak M, Li H, Allen K, et al. Programmable probiotics for detection of cancer in urine. Science translational medicine. 2015;7(289):289ra284.

14. Cronin M, Akin AR, Collins SA, Meganck J, Kim JB, Baban CK, et al. High resolution in vivo bioluminescent imaging for the study of bacterial tumour targeting. PloS one. 2012;7(1):e30940.

15. Sharma P, Hu-Lieskovan S, Wargo JA, Ribas A. Primary, Adaptive, and Acquired Resistance to Cancer Immunotherapy. Cell. 2017;168(4):707-23.

16. Rosenberg SA, Spiess PJ, Kleiner DE. Antitumor effects in mice of the intravenous injection of attenuated Salmonella typhimurium. Journal of immunotherapy. 2002;25(3):218-25.

17. Roberts NJ, Zhang L, Janku F, Collins A, Bai RY, Staedtke V, et al. Intratumoral injection of Clostridium novyi-NT spores induces antitumor responses. Science translational medicine. 2014;6(249):249ra111.

18. Yazawa K, Fujimori M, Amano J, Kano Y, Taniguchi S. Bifidobacterium longum as a delivery system for cancer gene therapy: selective localization and growth in hypoxic tumors. Cancer Gene Ther. 2000;7:269-74.

19. Brockstedt DG, Giedlin MA, Leong ML, Bahjat KS, Gao Y, Luckett W, et al. Listeria-based cancer vaccines that segregate immunogenicity from toxicity. Proc Natl Acad Sci USA. 2004;101(38):13832-7.

20. Toso JF, Gill VJ, Hwu P, Marincola FM, Restifo NP, Schwartzentruber DJ, et al. Phase I study of the intravenous administration of attenuated Salmonella typhimurium to patients with metastatic 
melanoma. Journal of clinical oncology: official journal of the American Society of Clinical Oncology. 2002;20(1):142-52.

21. Low KB, Ittensohn M, Le T, Platt J, Sodi S, Amoss M, et al. Lipid A mutant Salmonella with suppressed virulence and TNFalpha induction retain tumor-targeting in vivo. Nature biotechnology. 1999;17(1):37-41.

22. Zhao M, Yang M, Li XM, Jiang P, Baranov E, Li S, et al. Tumor-targeting bacterial therapy with amino acid auxotrophs of GFP-expressing Salmonella typhimurium. Proc Natl Acad Sci USA. 2005;102(3):755-60.

23. Loeffler M, Le'Negrate G, Krajewska M, Reed JC. Attenuated Salmonella engineered to produce human cytokine LIGHT inhibit tumor growth. Proc Natl Acad Sci USA. 2007;104(31):12879-83.

24. Rembacken BJ, Snelling AM, Hawkey PM, Chalmers DM, Axon AT. Non-pathogenic Escherichia coli versus mesalazine for the treatment of ulcerative colitis: a randomised trial. Lancet. 1999;354(9179):635-9.

25. Zhang Y, Ji W, He L, Chen Y, Ding X, Sun Y, et al. E. coli Nissle 1917-Derived Minicells for Targeted Delivery of Chemotherapeutic Drug to Hypoxic Regions for Cancer Therapy. Theranostics. 2018;8(6):1690-705.

26. Zhang Y, Zhang Y, Xia L, Zhang X, Ding X, Yan F, et al. Escherichia coli Nissle 1917 targets and restrains mouse B16 melanoma and 4T1 breast tumors through expression of azurin protein. Appl Environ Microbiol. 2012;78(21):7603-10.

27. He L, Yang H, Liu F, Chen Y, Tang J, Liu Z. Escherichia coli Nissle 1917 engineered to express Tum-5 can restrain murine melanoma growth. Oncotarget. 2017;8(49):85772-82.

28. He L, Yang H, Tang J, Liu Z, Chen Y, Lu B, et al. Intestinal probiotics E. coli Nissle 1917 as a targeted vehicle for delivery of p53 and Tum- 5 to solid tumors for cancer therapy. Journal of biological engineering. 2019;13:58.

29. SA R. The development of new immunotherapies for the treatment of cancer using interleukin-2. A review. 1988;208:121-35.

30. Klapper JA, Downey SG, Smith FO, Yang JC, Hughes MS, Kammula US, et al. High-dose interleukin-2 for the treatment of metastatic renal cell carcinoma: a retrospective analysis of response and survival in patients treated in the surgery branch at the National Cancer Institute between 1986 and 2006. Cancer. 2008;113(2):293-301.

31. Rosalia RA, Arenas-Ramirez N, Bouchaud G, Raeber ME, Boyman O. Use of enhanced interleukin-2 formulations for improved immunotherapy against cancer. Curr Opin Chem Biol. 2014;23:39-46.

32. Hu P, Mizokami M, Ruoff G, Khawli LA, Epstein AL. Generation of low-toxicity interleukin-2 fusion proteins devoid of vasopermeability activity. Blood. 2003;101(12):4853-61.

33. Vazquez-Lombardi R, Loetsch C, Zinkl D, Jackson J, Schofield P, Deenick EK, et al. Potent antitumour activity of interleukin-2-Fc fusion proteins requires Fc-mediated depletion of regulatory T-cells. Nature communications. 2017;8:15373. 
34. Levin AM, Bates DL, Ring AM, Krieg C, Lin JT, Su L, et al. Exploiting a natural conformational switch to engineer an interleukin-2 'superkine'. Nature. 2012;484(7395):529-33.

35. Lazear E, Ghasemi R, Hein SM, Westwick J, Watkins D, Fremont DH, et al. Targeting of IL-2 to cytotoxic lymphocytes as an improved method of cytokine-driven immunotherapy. Oncoimmunology. 2017;6(2):e1265721.

36. Chen X, Ai X, Wu C, Wang H, Zeng G, Yang P, et al. A novel human IL-2 mutein with minimal systemic toxicity exerts greater antitumor efficacy than wild-type IL-2. Cell death disease. 2018;9(10):989.

37. Smith KA. Lowest dose interleukin-2 immunotherapy. Blood. 1993;81(6):1414-23.

38. Saltzman DA, Katsanis E, Heise CP, Hasz DE, Kelly SM, Curtiss R 3. Patterns of hepatic and splenic colonization by an attenuated strain of Salmonella typhimurium containing the gene for human interleukin-2: a novel anti-tumor agent. Cancer Biother Radiopharm. 1997;12(1):37-45. rd et al.

39. Sorenson BS, Banton KL, Frykman NL, Leonard AS, Saltzman DA. Attenuated Salmonella typhimurium with IL-2 gene reduces pulmonary metastases in murine osteosarcoma. Clin Orthop Relat Res. 2008;466(6):1285-91.

\section{Figures}




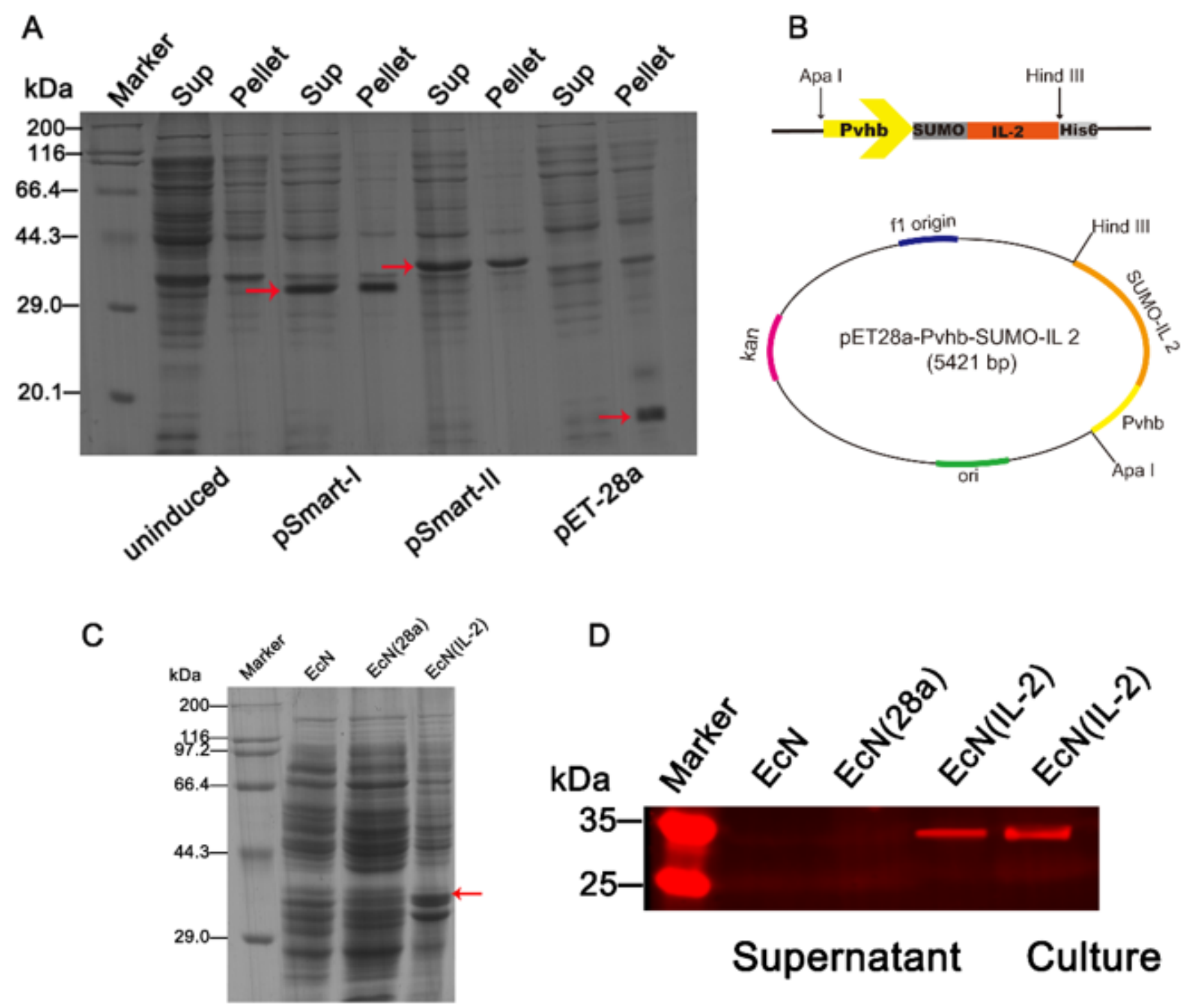

Figure 1

Characterization of IL-2 expression in vitro. (A) Coomassie Brilliant Blue staining of an SDS-PAGE gel showing IL-2 expression in E. coli BL21 (DE3). (B) Map of the recombination plasmid containing the IL-2 gene. (C) SDS-PAGE analysis of IL-2 expression in EcN, EcN (28a), and EcN (hIL-2). (D) Western blot analysis of IL-2 expression in cell lysates (supernatants) or culture supernatants of EcN, EcN (28a), and EcN (hIL-2). 

A
-

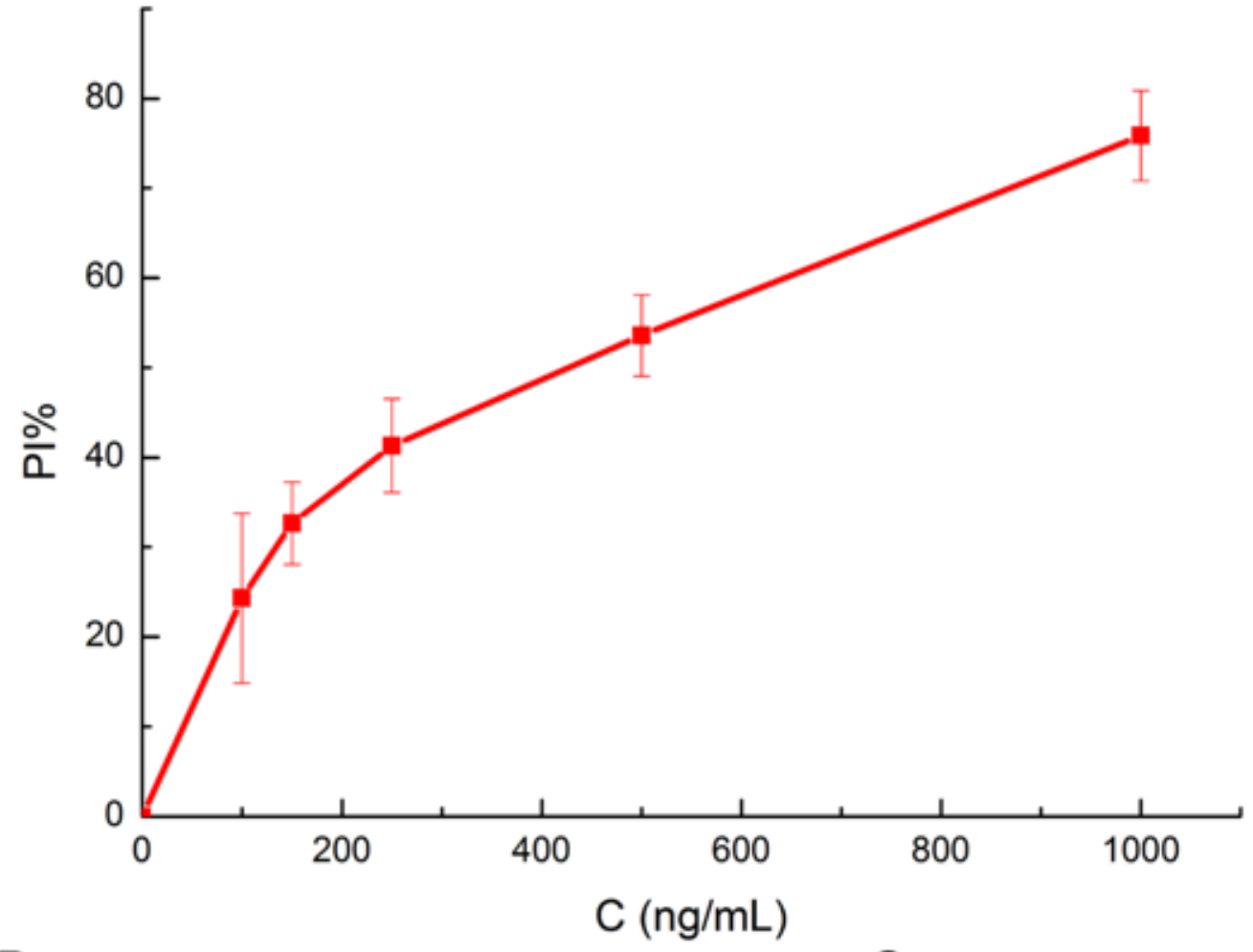

B

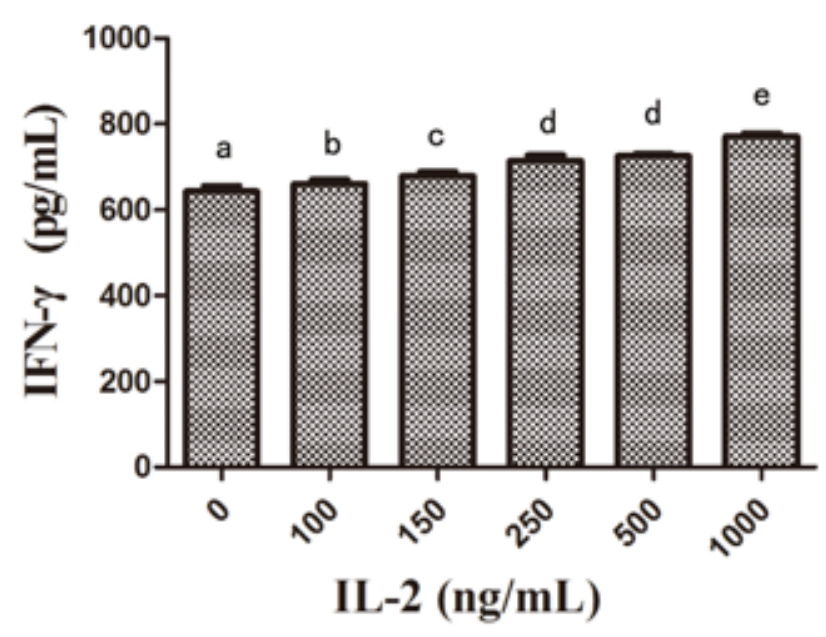

C

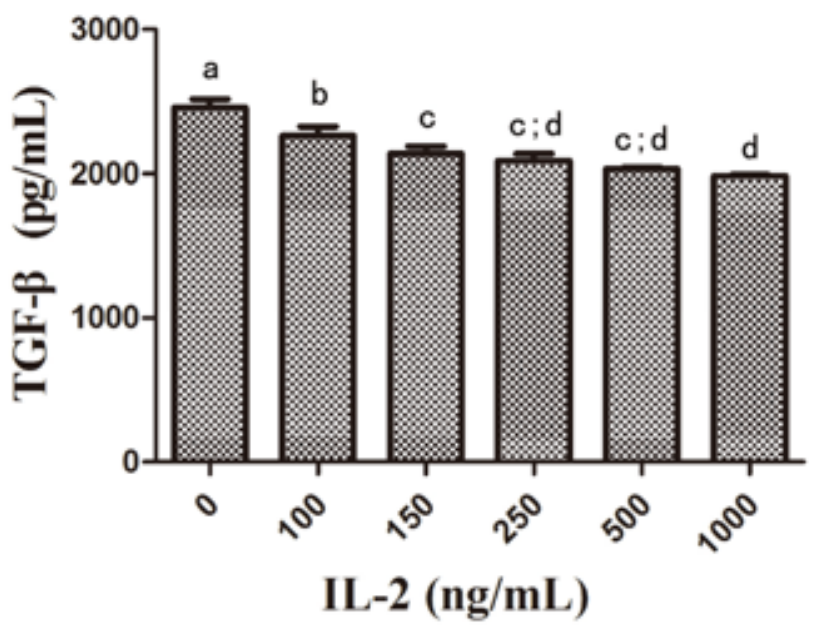

Figure 2

SUMO-IL-2 protein promotes the proliferation of PBMCs. The SUMO-IL-2 protein was divided into 5 different concentrations and incubated with PBMCs for $24 \mathrm{~h}$ before cell viability was detected. (A) A CCK8 kit was used to detect cell viability after the incubation. ELISA was used to detect the concentrations of IFN- $\gamma(B)$ and TGF- $\beta$ (B) in the culture supernatant. Different letters indicate significant differences (P $<0.05)$; the same letters indicate no significant difference. 
A

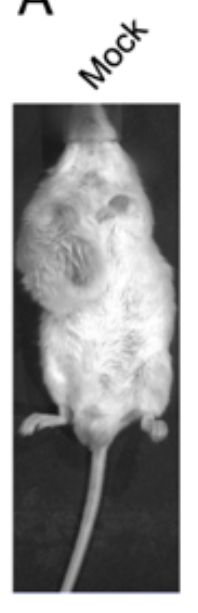

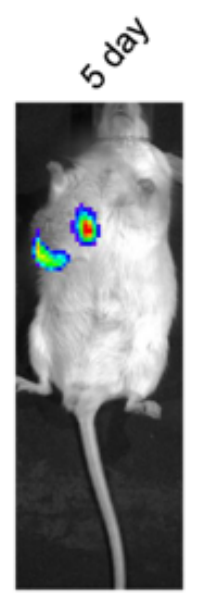

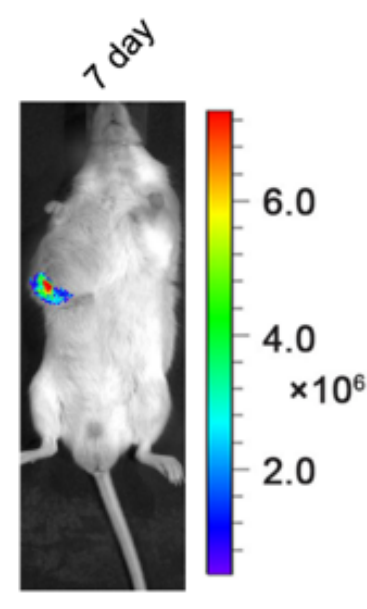

B

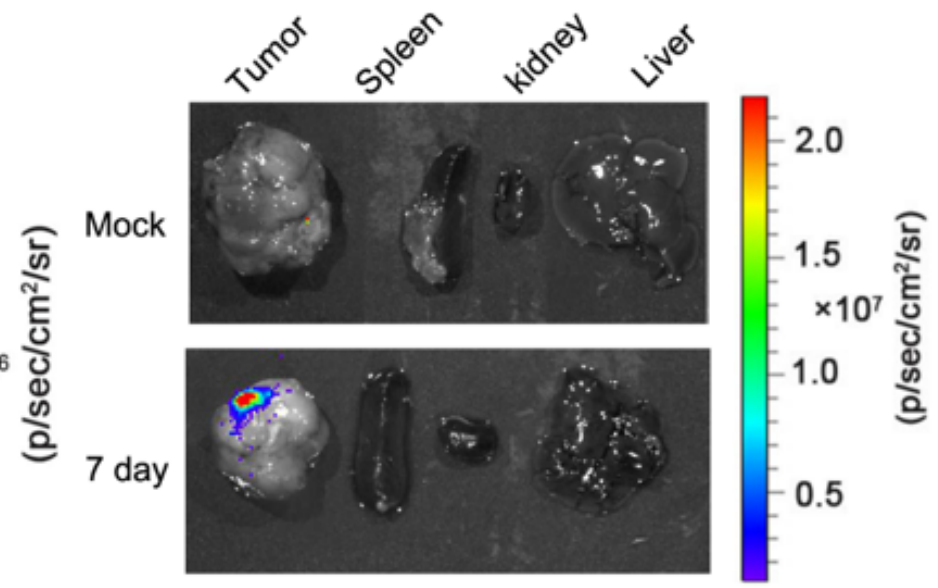

\section{Figure 3}

Colonization of a CT26 tumour model mice with EcN. CT26 cancer cells were inoculated subcutaneously in the right axilla of BALB/c mice, and 7 days later, the mice were i.p. injected with $5 \times 106 \mathrm{CFU} / 100 \mu \mathrm{L}$ $\mathrm{ECN}$ (Lux). (A) An IVIS was used to detect the distribution of EcN in tumour-bearing mice at different time points. (B) Liver, kidneys and spleen of mice in the two groups of mice were isolated and observed using the IVIS. 

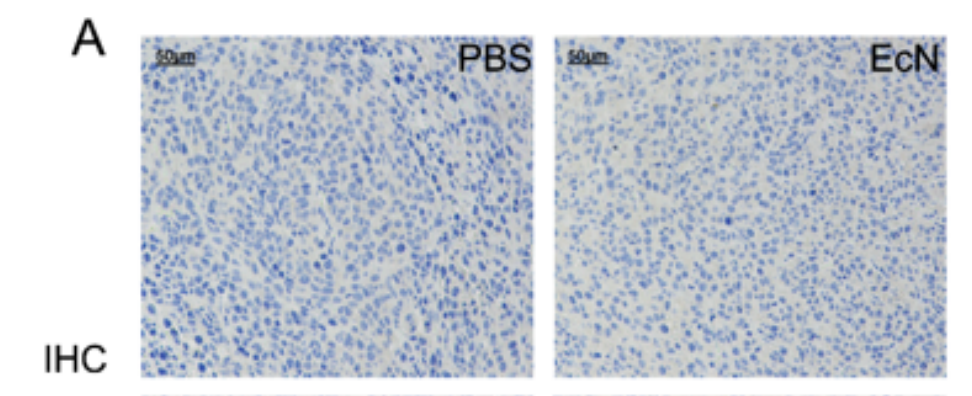

B

$(200 X)$
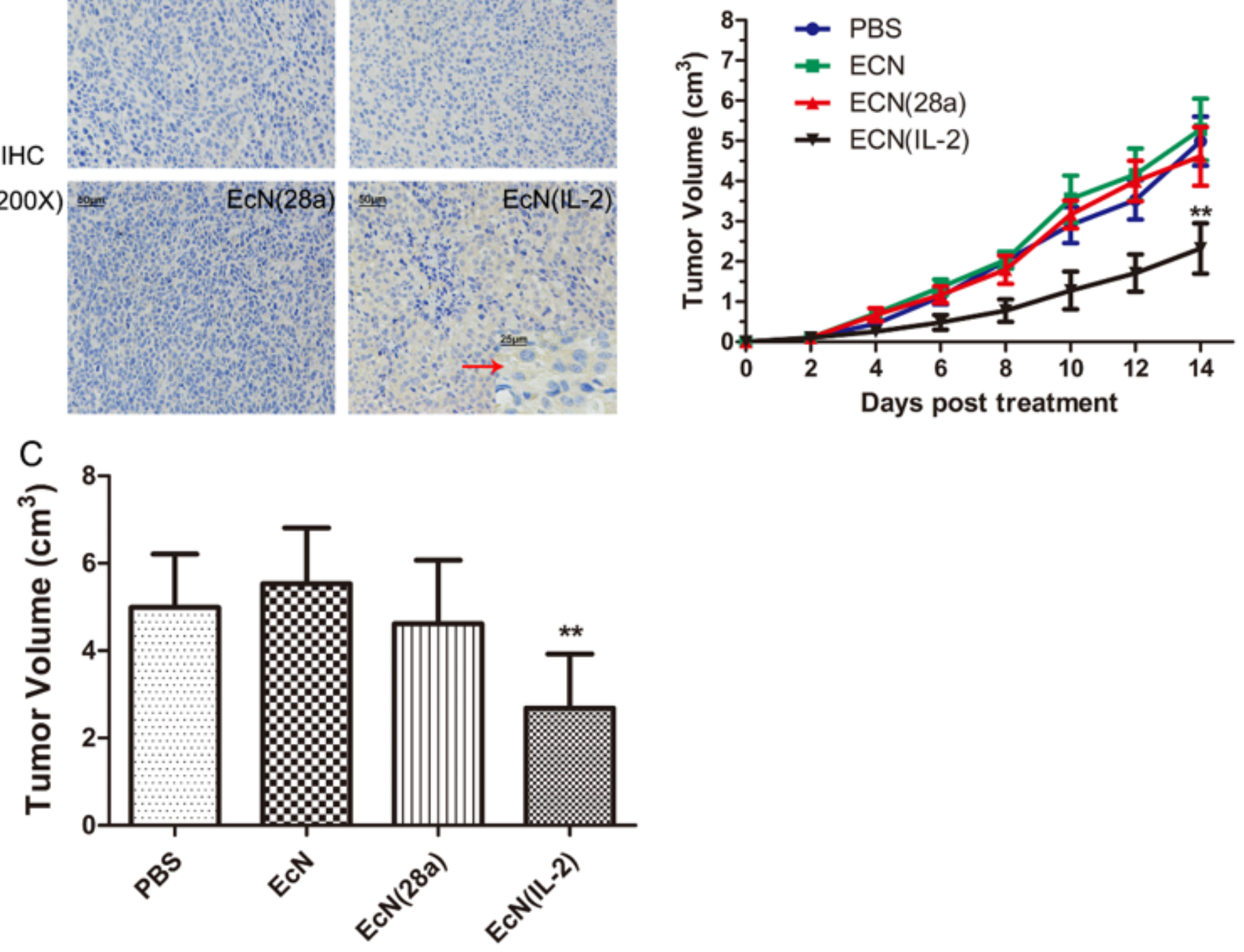

Figure 4

Stable expression of EcN (hIL-2) in tumour regions and its inhibition of CT26 colon tumours. (A) Successful expression of IL-2 in tumours was verified by IHC (200x). The red arrow refers to positive staining (400x). The therapeutic effect of EcN (hIL-2) on CT26 tumour-bearing mice was examined. (B) PBS, EcN, EcN (28a) or EcN (hIL-2) was injected i.p. into mice (bacterial injections contained $5 \times 106$ $\mathrm{CFU} / 100 \mu \mathrm{L}$ ), and tumour volumes (cm 3) were estimated using external callipers (values are expressed as the mean \pm standard deviation [SD]). (C) Tumours in the $\mathrm{EcN}$ (hIL-2)-treated group were significantly smaller than those in the other three groups. After the mice were sacrificed, their tumours were dissected and weighed; * $\mathrm{P}<0.05$, ** $\mathrm{P}<0.01$. 


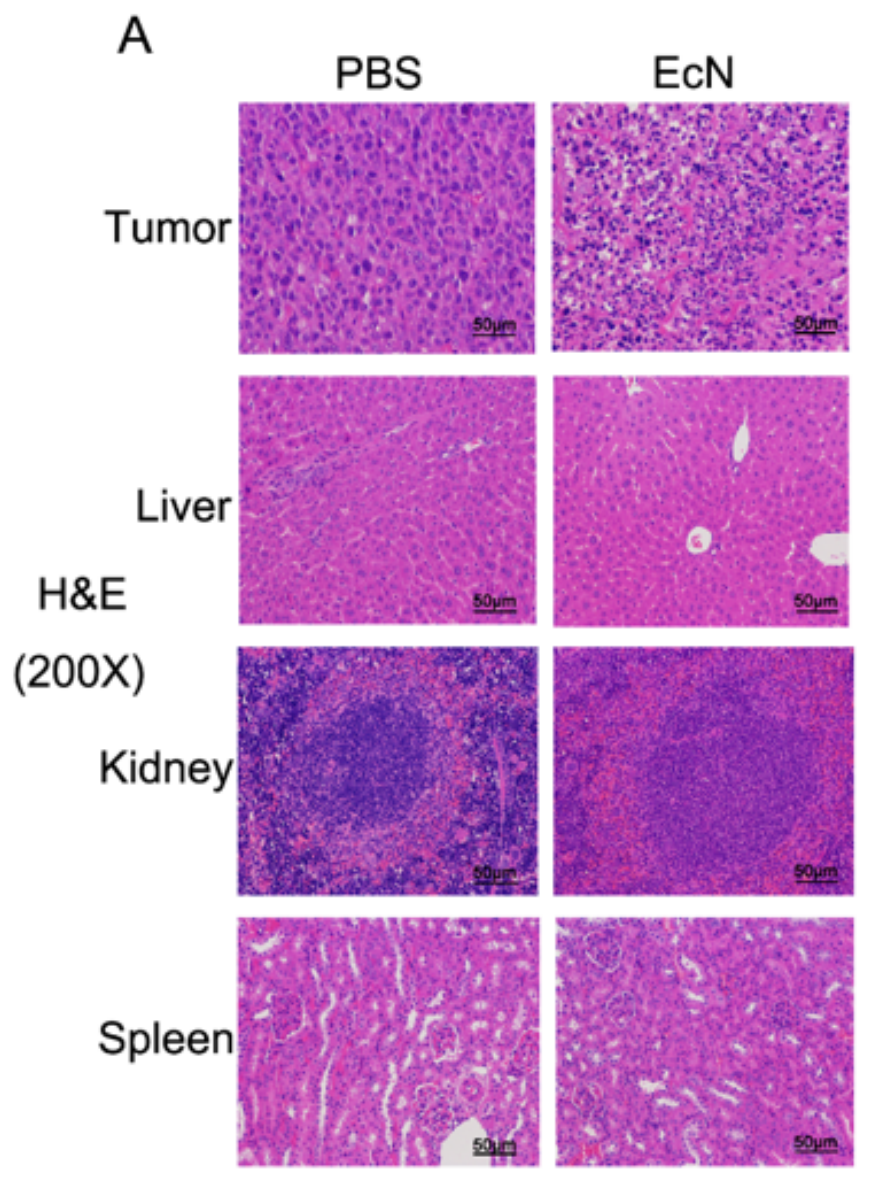

B
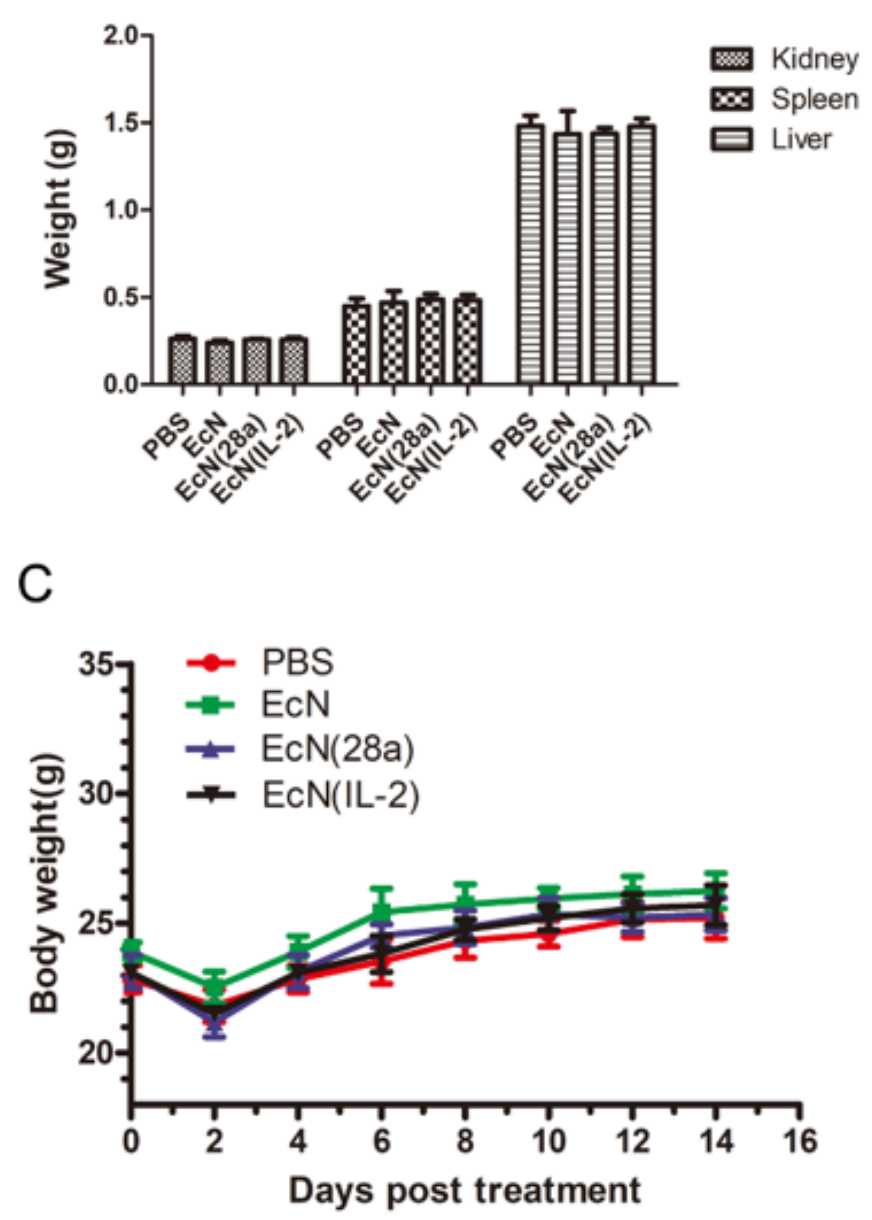

\section{Figure 5}

H\&E staining of tumour tissue sections and the effect of ECN on the liver, kidneys, spleen and body weight of mice. (A) H\&E staining (200x) of tumour tissue sections was used to observe an invasive inflammatory phenomenon in tumour tissue. H\&E staining (200x) of the liver, kidneys and spleen was used to observe the pathological morphology of the biopsies. (B) After mice in the four groups were sacrificed, the liver, kidneys and spleen of the mice were weighed (shown as the mean and SD). (C) Mouse body weights were recorded every two days during the experiment. No significant differences were observed among the four groups of mice. 

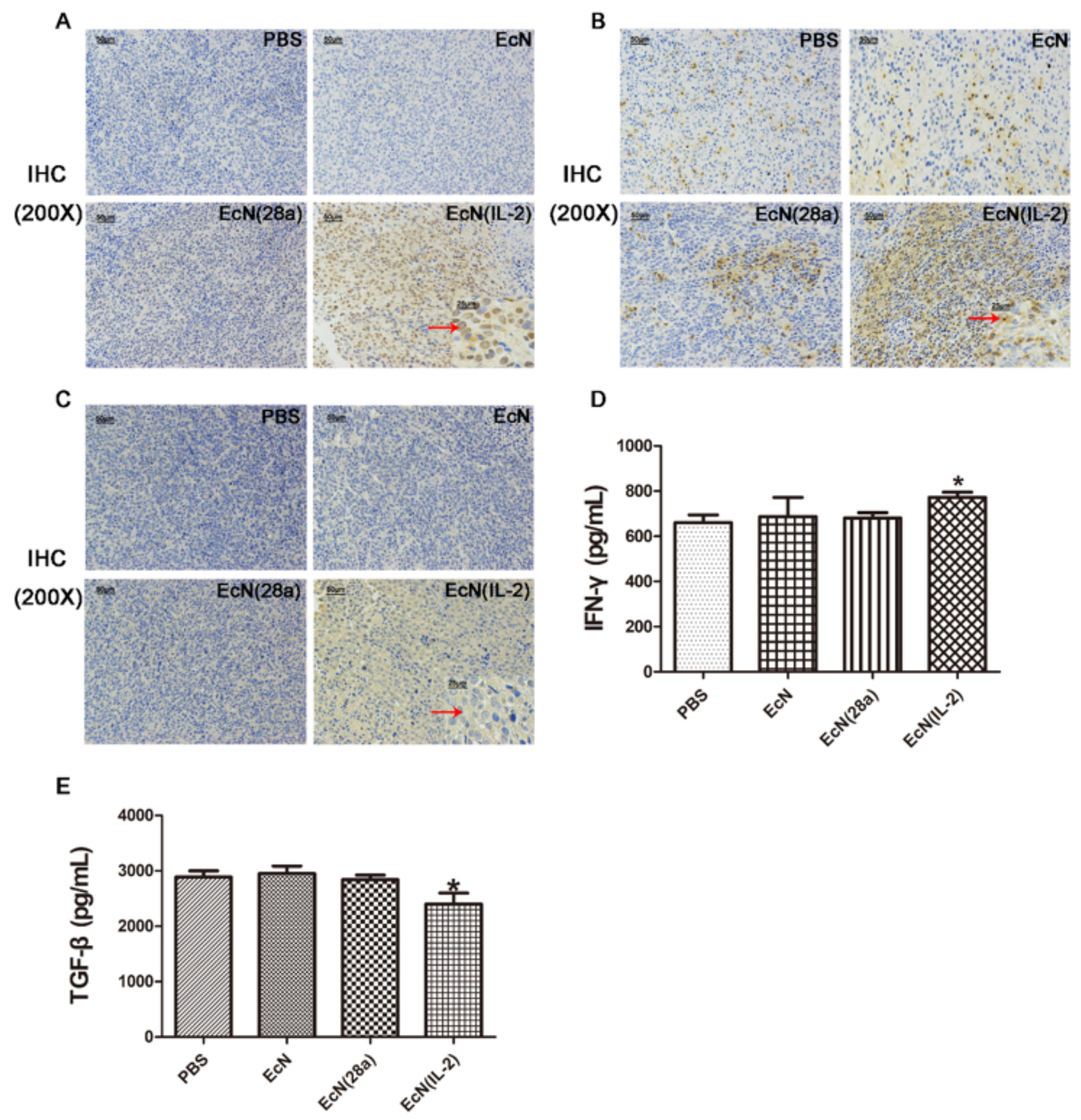

Figure 6

Immune status change in the tumour microenvironment post-bacterial treatments. In total, $1 \times 105$ CT26 cancer cells were inoculated subcutaneously into the right axilla of BALB/c mice, which were then treated with PBS, EcN, EcN (28a) or EcN (hIL-2) at 7 days post-tumour inoculation. At the end of the experiment, the mice were sacrificed, and tumours were collected to determine T lymphocyte (A), neutrophil (B) and M1 macrophage (C) levels by immunohistochemistry. The red arrow refers to positive staining (400x). 
Blood was collected from mice, and the tumour microenvironment was assessed by measuring INF- $Y$ and TGF- $\beta$ levels in the serum by ELISA (D, E); * $P<0.05$.

\section{Supplementary Files}

This is a list of supplementary files associated with this preprint. Click to download.

- Supplementarylnformation.pdf 